\title{
Breast carcinoma metastasis in a resected meningioma with early diagnosis of oligometastatic disease: a case report
}

\author{
Christian Fernandez ${ }^{1}$, Louis Cappelli ${ }^{2}$, Sara Chapin ${ }^{3}$, Lawrence Kenyon ${ }^{3}$, Christopher J. Farrell ${ }^{4}$, \\ Wenyin Shi ${ }^{1}$ \\ ${ }^{1}$ Department of Radiation Oncology, Thomas Jefferson University, Philadelphia, PA, USA; ${ }^{2}$ School of Osteopathic Medicine, Rowan University, \\ Stratford, NJ, USA; ${ }^{3}$ Department of Pathology Anatomy \& Cell Biology, Thomas Jefferson University, Philadelphia, PA, USA; ${ }^{4}$ Department of \\ Neurological Surgery, Thomas Jefferson University, Philadelphia, PA, USA \\ Correspondence to: Christian Fernandez, MD. Department of Radiation Oncology, Thomas Jefferson University, 111 S. 11th Street, Philadelphia, PA \\ 19107, USA. Email: christian.fernandez@jefferson.edu.
}

\begin{abstract}
Tumor-to-tumor metastasis describes the ability of primary tumors to metastasize to other primary tumors. These events generally occur in aggressive and widely-metastatic disease, with the appropriate management and significance of these events unknown. A 56-year-old woman with a history of bilateral, localized, invasive lobular breast carcinoma treated with surgery, systemic therapy, and adjuvant radiation presented five and two years post-treatment with progressive neurological symptoms. Imaging revealed an intracranial meningioma, and the patient underwent resection. Pathology revealed metastatic invasive lobular carcinoma cells within the resected meningioma, and the patient was treated with postoperative radiation without sequelae. Subsequent staging scans revealed a single osseous lesion suggestive of oligometastatic disease, and the patient was promptly started on systemic therapy.
\end{abstract}

Keywords: Case report; meningioma; breast neoplasms; neoplasm metastasis

Submitted Mar 20, 2020. Accepted for publication Sep 15, 2020.

doi: $10.21037 /$ cco-20-122

View this article at: http://dx.doi.org/10.21037/cco-20-122

\section{Introduction}

Tumor-to-intracranial tumor metastasis is a known but rare phenomenon, with relatively limited representation in the literature (1). This scenario is most likely to be witnessed with indolent tumors being seeded by an aggressive or widely metastatic carcinoma (2). The most common intracranial tumors to receive distant metastases are meningiomas, with breast carcinoma being the most common donor (3). The incidence of this occurrence is likely underreported, given the limited ability to evaluate beyond histopathologic confirmation, the high threshold to pursue neurosurgical intervention in asymptomatic patients, and increasing survival times with modern systemic therapies (2). The significance or mechanism of this process is not completely understood. In this study, we present the following case of a 56-year-old woman with an intracranial meningioma found to be seeded by previously-treated breast cancer primaries leading to the diagnosis of oligometastatic disease.

We present the following case in accordance with the CARE reporting checklist (available at http://dx.doi. org/10.21037/cco-20-122) (4).

\section{Case presentation}

The authors are accountable for all aspects of the work in ensuring that questions related to the accuracy or integrity of any part of the work are appropriately investigated and resolved. All procedures performed in studies involving human participants were in accordance with the ethical standards of the institutional and/or national research committee(s) and with the Helsinki Declaration (as revised in 2013).

A 56-year-old female patient presented with left lower extremity weakness and focal motor seizures. Evaluation revealed bilateral diplopia for the preceding two months and headaches for the past week. Her past medical history was 


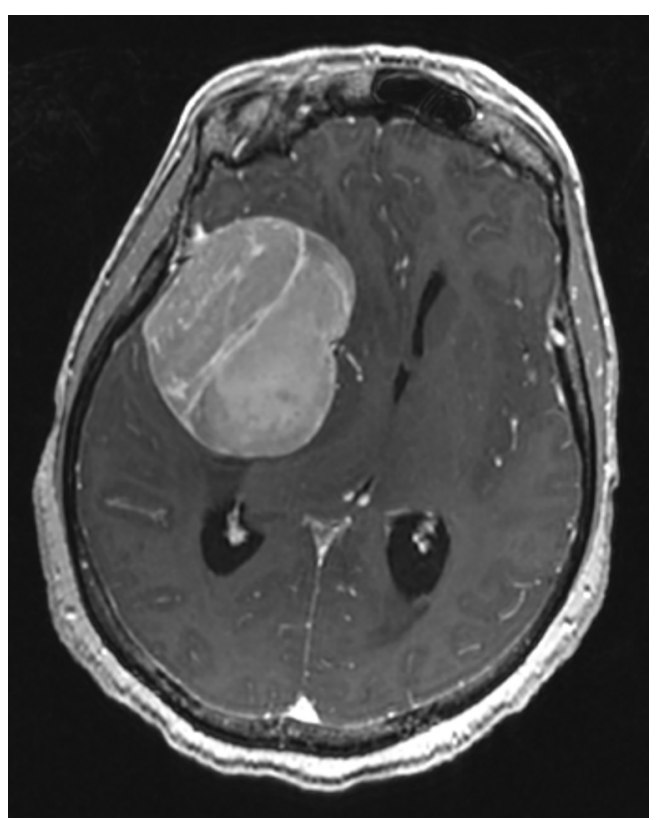

Figure $1 \mathrm{MRI}$ brain at presentation. Preoperative T1-weighted post-gadolinium sequence MRI of the brain in the axial plane demonstrating a $4.9 \mathrm{~cm} \times 6.5 \mathrm{~cm} \times 5.4 \mathrm{~cm}$ homogenouslyenhancing, intracranial mass involving the right greater wing of the sphenoid with midline shift.

remarkable for bilateral invasive lobular breast carcinoma, estrogen receptor (ER) and progesterone receptor (PR) positive, HER2-negative, both treated with surgery, chemotherapy, radiation, and hormonal therapy five years (right breast T3N1M0) and two years (left breast T1cN0M0) prior to presentation, respectively. Magnetic resonance imaging (MRI) of the brain revealed a large, extra-axial, homogenously-enhancing mass in the right middle cranial fossa, measuring $4.9 \mathrm{~cm} \times 6.5 \mathrm{~cm} \times 5.4 \mathrm{~cm}$ (Figure 1), with $1.4 \mathrm{~cm}$ midline shift and mild bowing of the sphenoid cortex into the orbit, consistent with meningioma. The patient underwent image-guided craniotomy with access through the root of the right zygoma. The mass was devascularized, sharp resection performed with extensive cauterization of the surrounding dura, and postoperative computed tomography (CT) of the head suggestive of gross total resection.

\section{Pathologic findings}

The final pathology report of the resected neoplasm revealed a World Health Organization (WHO) grade I meningioma (Figure $2 A$ ) with a Ki-67 proliferation index of
6.4\%. Due to some morphologic overlap with schwannoma, confirmatory immunohistochemical stains were performed. Immunohistochemistry for S100 was negative, while EMA showed mild diffuse immunoreactivity supportive of the diagnosis of meningioma (Figure 2B). However, there were rare individual and small clusters of darker-staining epithelioid cells within the meningioma that were not otherwise appreciated on the original hematoxylin and eosin (H\&E) stained sections (Figure 2C). By careful alignment of the EMA-stained cell clusters with the H\&E-stained sections, small linear clusters could be identified that were morphologically consistent with lobular breast carcinoma. Breast markers GATA3 and GCDFP-mammaglobin (Figure $2 D, E)$ confirmed the presence of metastatic lobular breast carcinoma. Additional immunohistochemistry for breast markers showed these tumor cells were positive for ER and 2+ HER2/Neu staining (Figure 2F). Dual in situ hybridization for HER2/Neu and PR staining were non-contributory due to the inability to reliably distinguish rare, scattered, breast carcinoma cells from meningioma cells, and diffuse meningioma PR positivity.

\section{Adjuvant treatment and follow up}

The patient was referred to radiation oncology for evaluation of adjuvant radiotherapy (RT) to the resection cavity. Given the presence of metastatic breast cancer, stereotactic body radiotherapy (SBRT) was recommended. MRI was obtained four months after resection, which revealed significant reduction in the resection cavity and a questionable, small residual meningeal nodularity in the posterior resection cavity on $\mathrm{T} 1$ post-gadolinium sequences. She was treated to a total dose of $30 \mathrm{~Gy}$ in five fractions using volumetric modulated arc therapy (VMAT) in three arcs, flattening-filter-free, prescribed to the $98.6 \%$ isodose line. She completed RT without acute toxicity, and subsequent complete resolution of presenting symptoms. PET scan one month after RT revealed a single, sclerotic, osseous lesion at L3, and she was referred to her medical oncologist and started on systemic therapy. MRI of the brain three and six months after RT revealed posttreatment changes without evidence of recurrence (Figure 3). Repeat staging scans revealed no other areas of disease. She continued to be without intracranial recurrence and received systemic therapy until three years after RT, when she suffered a myocardial infarction and expired. A timeline of care received by the patient is available in Figure 4 . 

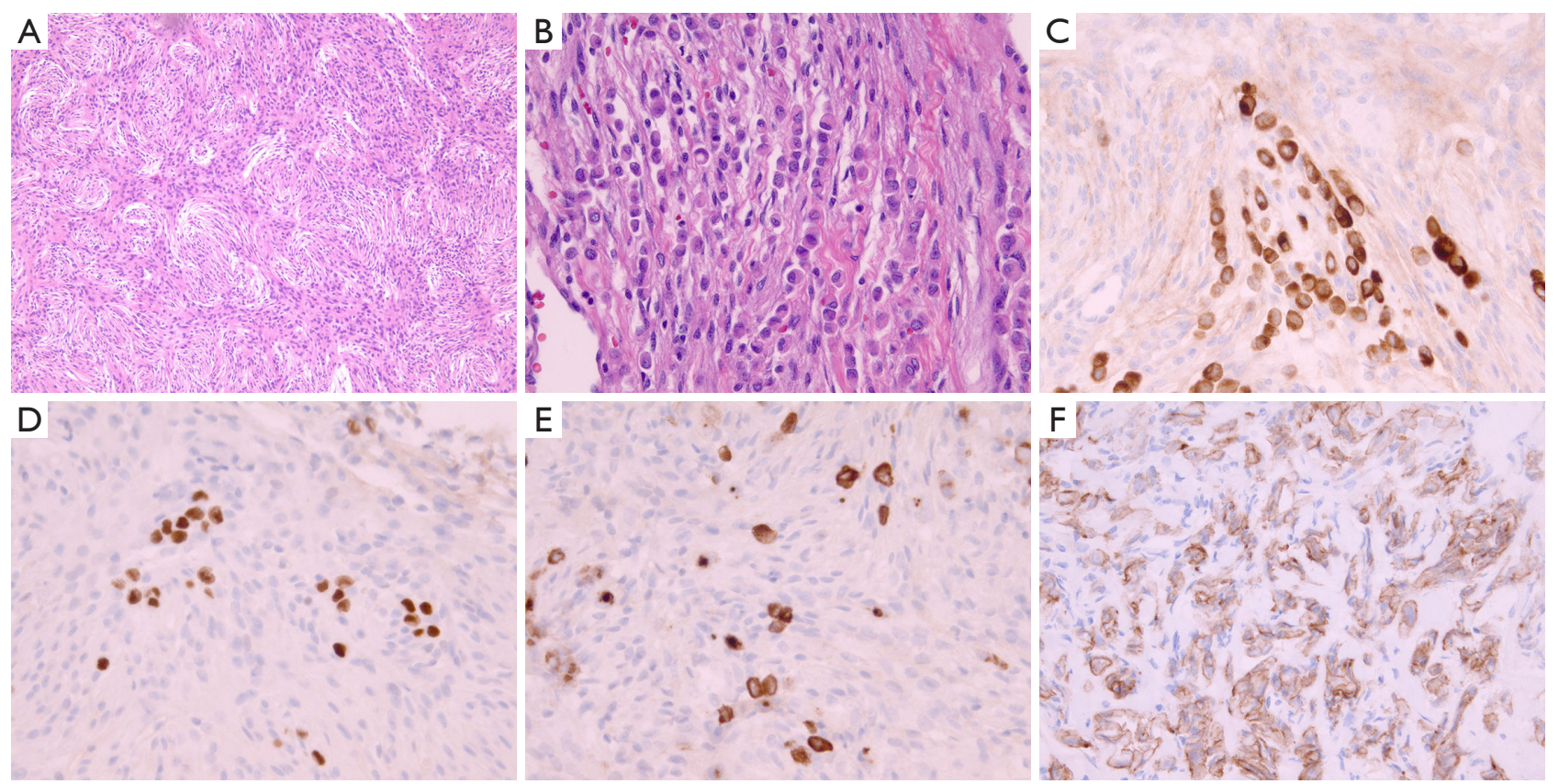

Figure 2 Meningioma pathology. Characteristic whorl pattern morphology of meningioma at 100× (A). EMA immunohistochemistry at $400 \times$ demonstrating diffuse background staining for the meningioma, along with darker staining lobular breast carcinoma cells (B). Singlefile pattern on $\mathrm{H} \& \mathrm{E}$ within the meningioma suggestive of lobular breast carcinoma at 400× (C). Lobular breast carcinoma cells at $400 \times$ showing immune-reactive for GATA3 (D), GCDFP-Mammoglobulin (E), and HER2Neu (F).

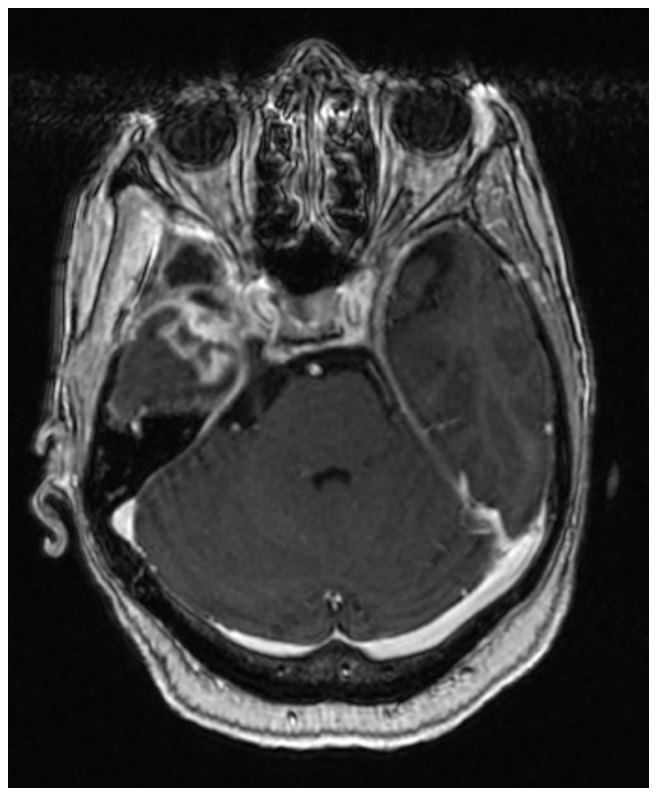

Figure 3 Post-treatment MRI brain. T1-weighted post-gadolinium sequence MRI of the brain six months post radiotherapy in the axial plane showing significant contraction of the surgical cavity, curvilinear enhancement, and stable meningeal enhancement along the craniotomy flap.

\section{Discussion}

In the 1930s, tumor-to-tumor metastasis to meningioma was first recorded by Fried BM (5). Of the total cases reported since, over $70 \%$ involved meningiomas, of which nearly half involved breast cancer metastasis (6). The prevalence of this phenomenon is postulated to be partly from the overlapping epidemiology of patients with breast cancer and meningiomas (7). In addition, the Swedish Cancer Registry and the United States Surveillance, Epidemiology, and End Results Program (SEER) reported an increase in the diagnosis of meningiomas after diagnosis of primary breast cancer, although this is likely a manifestation of increased brain imaging (8). Meningiomas are mostly slow-growing, hypervascular intracranial tumors. Such characteristics increase the chances of meningeal seeding via hematogenous spread. They have high lipid and collagen contents and lack a reactive immune response against foreign bodies, creating an optimal seeding environment (2,9-11). These factors may explain why meningiomas are the most common benign intracranial tumor to accept distant metastases (12).

The relatively increased affinity of metastatic breast carcinoma cells to meningiomas may also be attributed to facilitating receptor environments. Estrogen and 


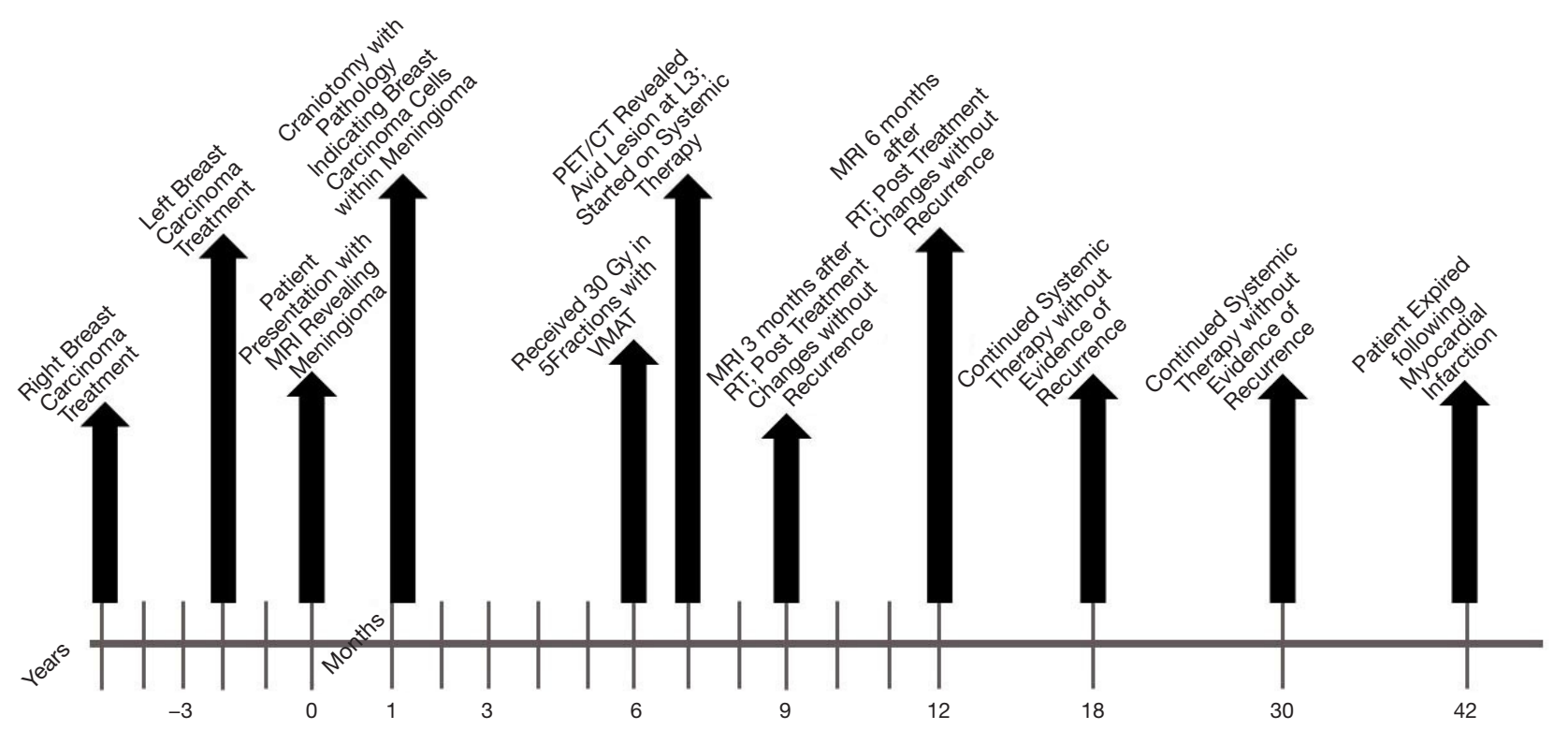

Figure 4 Timeline of events. Timeline of care received by the patient including relevant radiographic imaging, surgeries, and radiotherapies. MRI, magnetic resonance imaging; VMAT, volumetric modulated arc therapy; PET/CT, positron emission tomography/computed tomography; RT, radiotherapy.

progesterone receptors found on meningiomas are thought to be involved in cell-to-cell interactions with breast carcinoma cells (13). Furthermore, it has been suggested that breast cancer metastasis is mediated through the endothelial growth factor receptor (EGFR) family pathway. HER2-positive breast carcinoma in particular may develop metastases through HER3 receptor and neuregulin 1 EGFRs $(2,14)$. The HER3-receptor pathway plays a role in the growth of HER2-positive breast carcinoma cells while neuregulin 1 is believed to be upregulated on meningioma cells (14). These described pathways could be relevant for the mechanism of metastasis in the case presented.

The prognostic impact of tumor-to-tumor metastasis remains unclear. Unique to our case was the diagnosis of metastatic disease from meningioma resection and oligometastatic disease burden upon further work-up. Postsurgical RT in the presented case demonstrated efficacy in local control. The patient continued to experience relief of symptoms following surgical resection and RT, and limited extracranial disease. This outcome is seemingly discordant to many of the similarly-reported cases. The majority of reviewed cases describing tumor-to-tumor metastasis from breast carcinoma to an intracranial meningioma report diffusely metastatic disease, worsening of presenting symptoms, poor local control with RT or systemic therapy, and eventual mortality, or do not mention outcomes $(2,5,7,9-12,14)$. This is likely due to the early diagnosis of oligometastatic disease, rather than heavily pretreated, widely-metastatic disease, as in other reports. Given the constellation of factors that facilitate breast-to-meningioma metastases, and low resection rates of meningiomas among metastatic breast cancer patients, it is questionable if the prognostic significance, if any, will ever be elucidated. Patients with prior history of malignancy, especially breast cancer, who undergo resection of meningiomas warrant close pathologic examination, even without history of metastatic disease, as demonstrated by our case report. Further publications of similar cases should provide detailed documentation regarding clinical factors, pathology, extracranial disease status, and prior treatments to best characterize this phenomenon and factors associated with their occurrence.

\section{Conclusions}

In conclusion, we present a case of tumor-to-tumor metastasis from invasive lobular breast carcinoma to meningioma that resulted in the early diagnosis of oligometastatic, breast cancer. Careful pathologic examination of resected meningiomas in this patient group is warranted. Future reports should include detailed information regarding 
patients, pathology, disease control, and treatment history.

\section{Acknowledgments}

We thank Kinga Skowron, MD, for her critical proof readings.

Funding: None.

\section{Footnote}

Reporting Checklist: The authors have completed the CARE reporting checklist. Available at http://dx.doi.org/10.21037/ cco-20-122

Conflicts of Interest: All authors have completed the ICMJE uniform disclosure form (available at http://dx.doi.org/10.21037/ cco-20-122). Dr. Shi reports grants from Novocure, grants from Regeneron, grants from North American Skull Base Society, personal fees from novocure, personal fees from brain lab, personal fees from Varian, personal fees from Zai, outside the submitted work. Dr. Shi serves as an unpaid editorial board member of Chinese Clinical Oncology. The other authors have no conflicts of interest to declare.

Etbical Statement: The authors are accountable for all aspects of the work in ensuring that questions related to the accuracy or integrity of any part of the work are appropriately investigated and resolved. All procedures performed in studies involving human participants were in accordance with the ethical standards of the institutional and/or national research committee(s) and with the Helsinki Declaration (as revised in 2013). Patient consent was obtained and information reported as per approved IRB \#18D.480.

Open Access Statement: This is an Open Access article distributed in accordance with the Creative Commons Attribution-NonCommercial-NoDerivs 4.0 International License (CC BY-NC-ND 4.0), which permits the noncommercial replication and distribution of the article with the strict proviso that no changes or edits are made and the original work is properly cited (including links to both the formal publication through the relevant DOI and the license). See: https://creativecommons.org/licenses/by-nc-nd/4.0/.

\section{References}

1. Takei H, Powell SZ. Tumor-to-tumor metastasis to the central nervous system. Neuropathology 2009;29:303-8.
2. Sayegh ET, Burch EA, Henderson GA, et al. Tumor-totumor metastasis: breast carcinoma to meningioma. J Clin Neurosci 2015;22:268-74.

3. Erdogan H, Aydin MV, Tasdemiroglu E. Tumor-to-tumor metastasis of the central nervous system. Turk Neurosurg 2014;24:151-62.

4. Riley DS, Barber MS, Kienle GS, et al. CARE guidelines for case reports: explanation and elaboration document. J Clin Epidemiol 2017;89:218-35.

5. Fried BM. Metastatic Inoculation of a Meningioma by Cancer Cells from a Bronchiogenic Carcinoma. Am J Pathol 1930;6:47-52.1.

6. Syed S, Karambizi DI, Baker A, et al. A Comparative Report on Intracranial Tumor-to-Tumor Metastasis and Collision Tumors. World Neurosurg 2018;116:454-463.e2.

7. Zon LI, Johns WD, Stomper PC, et al. Breast carcinoma metastatic to a meningioma. Case report and review of the literature. Arch Intern Med 1989;149:959-62.

8. Ahsan H, Neugut AI, Bruce JN. Association of malignant brain tumors and cancers of other sites. J Clin Oncol 1995;13:2931-5.

9. Sayegh ET, Henderson GA, Burch EA, et al. Intrameningioma metastasis of breast carcinoma. Rare Tumors 2014;6:5313.

10. Neville IS, Solla DF, Oliveira AM, et al. Suspected tumorto-meningioma metastasis: A case report. Oncol Lett 2017;13:1529-34.

11. Abrahão-Machado L, Abrahao-Machado E, AbrahaoMachado E, et al. Tumor-To-Tumor Metastasis: Intracranial Meningioma Harboring Metastatic Breast Carcinoma. Ann Clin Pathol 2015;3:1049.

12. Caroli E, Salvati M, Giangaspero F, et al. Intrameningioma metastasis as first clinical manifestation of occult primary breast carcinoma. Neurosurg Rev 2006;29:49-54.

13. Pham JT, Kim RC, Nguyen A, et al. Intracranial meningioma with carcinoma tumor-to-tumor metastasis: two case reports. CNS Oncol 2018;7:CNS09.

14. McCormack M, Rosa CSL, Murphy M, et al. HER2positive breast cancer metastatic to intracranial meningioma: a case report. J Clin Pathol 2013;66:633-4.

Cite this article as: Fernandez C, Cappelli L, Chapin S, Kenyon L, Farrell CJ, Shi W. Breast carcinoma metastasis in a resected meningioma with early diagnosis of oligometastatic disease: a case report. Chin Clin Oncol 2020;9(5):71. doi: $10.21037 /$ cco-20-122 